\title{
Functional Characterization of the NF- $\mathrm{B}$ p65 Transcriptional Activator and an Alternatively Spliced Derivative
}

\author{
STEVEN M. RUBEN, ${ }^{1}$ RAMASWAMY NARAYANAN,${ }^{2}$ JOHN F. KLEMENT,${ }^{1}$ CHIEN-HWA CHEN,${ }^{1}$ \\ AND CRAIG A. ROSEN ${ }^{1 *}$ \\ Department of Gene Regulation, Roche Institute of Molecular Biology, ${ }^{1}$ and Department of Molecular Genetics, \\ Hoffmann La-Roche, Inc., ${ }^{2} 340$ Kingsland Street, Nutley, New Jersey 07110-1199
}

Received 28 August 1991/Accepted 30 October 1991

\begin{abstract}
The NF-kB transcription factor complex is composed of two proteins, designated p50 and p65, both having considerable homology to the product of the rel oncogene. We present evidence that the p65 subunit is a potent transcriptional activator in the apparent absence of the p50 subunit, consistent with in vitro results demonstrating that p65 can interact with DNA on its own. To identify the minimal activation domain, chimeric fusion proteins between the DNA binding domain of the yeast transcriptional activator protein GAL4 and regions of the carboxy terminus of p65 were constructed, and their transcriptional activity was assessed by using a GAL4 upstream activation sequence-driven promoter-chloramphenicol acetyltransferase fusion. This analysis suggests that the boundaries of the activation domain lie between amino acids 415 and 550 . Moreover, single amino acid changes within residues 435 to 459 greatly diminished activation. Similar to other activation domains, this region contains a leucine zipper-like motif as well as an overall net negative charge. To identify those residues essential for DNA binding, we made use of a naturally occurring derivative of p65, lacking residues 222 to 231 (hereafter referred to as p65 $\Delta$ ), and produced via an alternative splice site. Gel mobility shift analysis using bacterially expressed p65, p65 $\Delta$, and various mutants indicates that residues 222 to 231 are important for binding to $\mathrm{kB}$ DNA. Coimmunoprecipitation analysis suggests that these residues likely contribute to the multimerization function required for homomeric complex formation or heteromeric complex formation with p50 in that no association of p65 $\Delta$ with itself or with p50 was evident. However, p65 $\Delta$ was able to form weak heteromeric complexes with p65 that were greatly reduced in their ability to bind DNA. On the basis of these findings, we suggest that subtle changes within the proposed multimerization domain can elicit different effects with the individual Rel-related proteins and that a potential role of p65 $\Delta$ may be to negatively regulate $\mathrm{NF}-\mathrm{kB}$ function through formation of nonfunctional heteromeric complexes.
\end{abstract}

The NF-kB transcription factor complex is a pleiotropic activator that participates in the induction of numerous cellular and viral genes $(4,32)$. The active complex is composed of two subunits, designated p50 and p65 $(3,18)$. The genes encoding p50 $(19,29)$ and p65 $(37,45)$ have recently been cloned, and the amino termini of both proteins have been shown to have considerable homology to the rel oncogene product. The rel oncogene, originally identified in the avian retrovirus Rev $\mathrm{T}$, causes lymphoid cell tumors in birds (41). It is now clear that the Rel family of proteins, of which the Drosophila maternal morphogen dorsal is a member (55), possess transcriptional regulatory properties $(8,16$, $23,28,42,58)$, as best exemplified by the NF- $\mathrm{kB}$ transcription factor complex.

NF- $\mathrm{kB}$ exists in both an active and an inactive state. The inactive complex is present in the cytosol as a complex bound to an inhibitor protein termed IкB $(1,2)$. The property of regulation by subcellular localization is shared by each of the Rel-related family members. For example, the chicken c-rel protein is localized in the cytoplasm of avian fibroblasts (10), whereas the v-rel protein is nuclear in these cells (10). In transformed avian lymphoid cells, v-rel is localized primarily in the cytoplasm (20). Similarly, the dorsal protein is localized in the cytoplasm of cleavage stage embryos, whereas in the blastoderm it is relocalized in a graded fashion to the ventral nuclei $(43,47,56)$. Translocation of

\footnotetext{
* Corresponding author.
}

NF-кB to the nucleus can be induced by a variety of stimuli, including viral proteins $(5,33,44)$, mitogens $(6,52)$, and several cytokines (38). Nuclear translocation is thought to reflect the dissociation of the IKB inhibitor from the p65 subunit, a process that likely requires phosphorylation of IкB (18).

The $\mathrm{p} 50$ product is processed from a $\mathrm{p} 105$ precursor which demonstrates no specific DNA binding capacity of its own $(19,29)$. The nonbinding $\mathrm{COOH}$-terminal processed region of p105 contains a repeat structure that exhibits extensive homology to ankyrin and several tissue differentiation and cell cycle control proteins. Similar ankyrin repeat-like elements are also present in IкB (24). The p65 subunit contains 550 amino acids $(37,45)$. A notable feature of $\mathrm{p} 65$, in addition to its extensive homology to c-rel, is the presence of a proline-rich carboxy terminus. Proline residues in this region account for $19 \%$ of the amino acids, which is twice that of the Rel-related region. Although the role of these residues in p65 function remains to be established, proline-rich regions have been identified in several other mammalian transcription factors, including CTF/NF-I (35), AP-2 (62), and c-Jun/ AP-1 (57).

From earlier studies, it was inferred that $\mathrm{p} 50$ confers the DNA binding specificity and that p65 confers the activation function of the NF-kB complex $(29,59)$. However, recent reports using both native $(60)$ and cloned $(37,45)$ p 65 protein indicate that, at least in vitro, it can associate with $\kappa \mathrm{B}$ DNA in the absence of p50. These findings raised the possibility 
that p65 functions as a transcriptional activator in the absence of association with p50.

In this study, we have identified specific domains within p65 that specify activation and DNA binding functions. We have found that $\mathrm{p} 65$ is a potent transcriptional activator of certain $\kappa B$ motifs in the apparent absence of p50 and that residues between 415 and 550 constitute the activation domain. Specifically, this region contains a leucine zipperlike motif (residues 435 to 459), which affects transcriptional activation when mutated, but it also contains an overall net negative charge, similar to other activation domains. We also identify a naturally occurring alternatively spliced derivative of p65, designated p65 $\Delta$, that lacks amino acids 222 to 231. Results obtained from gel mobility shift analysis using purified p65 and p65 6 and coimmunoprecipitation analysis using in vitro-translated proteins indicate that residues 222 to 231 contribute to the formation of homodimers and heterodimers with p50. p65 $\Delta$, which lacks residues 222 to 231 , could only form weak heteromeric complexes with p65 that either have a greatly reduced ability to bind DNA or are unable to bind DNA. The observation that these residues can affect both multimerization as well as DNA binding properties raises the possibility that residues which contribute to multimerization coincide with or lie close to the domain that specifies DNA binding.

\section{MATERIALS AND METHODS}

PCR analysis. Total RNA was prepared from approximately $10^{7}$ cells by using RNAzol (CINNA/Biotex, Houston, Tex.). First-strand cDNA was prepared from $1 \mu \mathrm{g}$ of total RNA, using oligo(dT) as a primer for RNase $\mathrm{H}^{-}$mouse mammary tumor virus reverse transcriptase (Bethesda $\mathrm{Re}$ search Laboratories, Gaithersburg, Md.). One microliter of cDNA was used as the template in polymerase chain reaction (PCR) analysis, using the two primers 5'-GCGGCC AAGCTTCAAGATCTGCCGAGTTG-3' and 5'-CGCTGC TCTAGAACACAATGGCCACTTGTCG-3', spanning the exons surrounding the p65 deletion. Amplification was for 35 cycles in a Perkin-Elmer thermocycler; each cycle consisted of denaturation at $94^{\circ} \mathrm{C}(1 \mathrm{~min})$, annealing at $55^{\circ} \mathrm{C}(2 \mathrm{~min})$, and extension at $72^{\circ} \mathrm{C}(3 \mathrm{~min})$. Reaction products were analyzed by gel electrophoresis on $3 \%$ agarose gels and visualized by staining with ethidium bromide.

For PCR analysis of mouse bone marrow cells, colonies (50 to 100 cells) were picked, and total RNA was extracted with $200 \mu \mathrm{l}$ of RNAzol in the presence of carrier tRNA (20 $\mu g)$. cDNA was reverse transcribed by using random hexamers, and PCR analysis of the cDNA was carried out as described above for 45 instead of 35 cycles. The integrity of the cDNA products was confirmed by Southern blot analysis (not shown). To examine the intron structure in the region of the p65 deletion, human CEM T-lymphocyte genomic DNA was amplified by using the same set of primers and thermocycler conditions. The amplified products, which were flanked by unique restriction sites, were cloned into a Bluescript plasmid vector (Stratagene, La Jolla, Calif.) and subjected to DNA sequencing (51).

Plasmid constructions. Eukaryotic expression vectors were constructed by placing either p65 or p65 $\Delta$ cDNA obtained from rescued phagemid (53) between a cytomegalovirus (CMV) promoter- $\beta$-globin intron and simian virus $\mathbf{4 0}$ poly(A) signal. The p50 cDNA was obtained by PCR amplification of Jurkat T-cell cDNA with primers specific to the p50 open reading frame corresponding to amino acids 1 to 502 of the p105 precursor protein (29). The resultant product was cloned into the same CMV promoter- $\beta$-globin intron expression vector. The $\mathrm{p} 50 / \mathrm{p} 65$ chimeric protein was generated by fusing the sequence encoding amino acids 1 to 370 of p50 to the sequence corresponding to amino acids 309 to 550 of p65, using PCR mutagenesis (25) followed by cloning into the above expression vector. The GAL4-p65 chimeric proteins were constructed by first amplifying the fragment of p65 corresponding to the indicated amino acids (see Fig. 5), restricting the amplified fragment with $B a m H I$ and $X b a I$, and cloning the fragments in frame with the GAL4 sequence corresponding to amino acids 1 to 147 of the GAL4 DNA binding domain in plasmid pSG424 (48). Mutagenesis of the leucine zipper motif was performed by PCR mutagenesis (25) of the p65 cDNA, using the same primers used for construction of the GAL 1-147/p65 295-550 chimeric gene or a primer at the $5^{\prime}$ terminus of p65 for construction of CMV-p65 L440/P and CMV-p65 L L442. Plasmids HkB-4 CAT and IL2R-kB-4 CAT contain four tandem copies of either the $\kappa B$ sequence from the human immunodeficiency virus type 1 (HIV-1) enhancer of interleukin-2 receptor $\alpha$ (IL-2R $\alpha)$ enhancer, respectively (33). Plasmid IL2R-421/-227 has been described previously (44). Epitope-tagged p65 and p65 genes were constructed by PCR, using a $5^{\prime}$ primer encoding the amino acid sequence MYPYDVPDYA corresponding to the influenza virus hemagglutinin protein (HA) (30). Plasmids p65 (1-309), p65 (1-299), and p50 (1-377) containing the residues necessary for DNA binding (1 to 377 for p50, 1 to 309 for p65, and 1 to 299 for p65 $)$ were constructed by PCR amplification of the corresponding cDNA, and the fragments were fused to a six-histidine moiety under control of a bacteriophage T5 promoter regulated by a lac operon (17). To construct $\mathrm{p} 50$, p65, and $\mathrm{p} 65 \Delta$ genes containing an influenza virus HA epitope tag, the respective cDNAs were cloned into a Bluescript (Stratagene) expression vector following, and in frame with, an oligonucleotide encoding the HA epitope tag (30). Plasmids encoding the truncated epitope-tagged p 65 and p $65 \Delta$ sequences were constructed by cloning the sequences encoding amino acids 1 to 309 and 1 to 299 for p65 and p65 , respectively, into the Bluescript vector encoding the HA tag.

Cell culture and transfection. Jurkat $\mathrm{T}$ cells were maintained in RPMI 1640 medium containing $10 \%$ fetal calf serum and $50 \mu \mathrm{g}$ of gentamicin (GIBCO, Bethesda, Md.) per ml. For transient transfection assays, $5 \times 10^{6}$ cells were transfected by the DEAE-dextran procedure (40). Cells were transfected with $2 \mu \mathrm{g}$ of reporter plasmid and 3 to $4 \mu \mathrm{g}$ of the p65 or p50 CMV expression vector. Cells were harvested 48 $\mathrm{h}$ after transfection, and chloramphenicol acetyltransferase (CAT) assays were performed as previously described (21). Cos 7 cells were maintained in Iscove modified Dulbecco medium plus $10 \%$ fetal calf serum supplemented with 4,500 $\mu \mathrm{g}$ of glucose per $\mathrm{ml}$. For transient transfection assays, $2 \times$ $10^{5}$ cells were plated on $35-\mathrm{mm}$ plates, and a modified DEAE-dextran protocol (12) was used to transfect the cells the following day. Cells were transfected with $1 \mu \mathrm{g}$ of the GAL4 upstream activation sequence (UAS) reporter construct and $2 \mu \mathrm{g}$ of the GAL4-p65 chimeric expression vectors.

In vitro transcription and translation and coimmunoprecipitation analysis. The $\mathrm{p} 50$, p65, or $\mathrm{p} 65 \Delta \mathrm{cDNA}$ present in the Bluescript expression vector was linearized with $B a m \mathrm{HI}$, and $2 \mu \mathrm{g}$ was used as the template for in vitro transcription with T7 RNA polymerase. The in vitro-transcribed RNA was used to program a wheat germ translation lysate (Promega, Madison, Wis.) that contained [ ${ }^{35}$ S]methionine. Protein products were analyzed on an $8 \%$ sodium dodecyl 
sulfate (SDS)-polyacrylamide gel and fluorographed. For the coimmunoprecipitation analysis, $1 \mu$ l of RNA corresponding to protein containing the HA tag was cotranslated with $1 \mu \mathrm{l}$ of RNA corresponding to either p65 or p50 lacking the tag sequence. Coimmunoprecipitation translations were carried out in a rabbit reticulocyte translation lysate (Promega). For immunoprecipitation, $4 \mu$ l of lysate was mixed with $150 \mu$ l of immunoprecipitation buffer ( $20 \mathrm{mM} N$-2-hydroxyethylpiperazine- $N^{\prime}$-2-ethanesulfonic acid [HEPES; pH 7.5], $250 \mathrm{mM}$ $\mathrm{NaCl}, 4 \mathrm{mM}$ EDTA, 0.1\% Nonidet P-40 [NP-40]). Two microliters of anti-HA immune serum (BAb Co, Berkeley, Calif.) was added to the reaction, after which protein $G$ agarose beads (Pharmacia, Piscataway, N.J.) were added. The samples were incubated at $4^{\circ} \mathrm{C}$ for $3 \mathrm{~h}$ and washed three times with immunoprecipitation buffer. Agarose beads were heated to $80^{\circ} \mathrm{C}$ for $10 \mathrm{~min}$ prior to gel loading. Protein products were analyzed on a $10 \%$ SDS-polyacrylamide gel and fluorographed.

Expression and purification of bacterially expressed proteins. A pDS expression plasmid was used for expression of p50, p65, and p65 in Escherichia coli (17). Briefly, expression was driven from a bacteriophage $\mathrm{T} 5$ promoter under control of a lac operator. Residues corresponding to the DNA binding domain of each protein (see above) were fused in frame with the six-histidine moiety present in plasmid pDS. Expression of these proteins was induced in mid-logarithmic-phase cultures of $E$. coli by addition of 1 $\mathrm{mM}$ isopropyl- $\beta$ - $D$-thiogalactopyranoside (IPTG). After a 4-h incubation, cells were pelleted and lysed in $6 \mathrm{M}$ guanidine hydrochloride $(\mathrm{pH} \mathrm{8.0)}$. The cleared lysate was adsorbed to a nickel chelate affinity resin, and proteins were eluted with a $\mathrm{pH}$ step gradient of $6 \mathrm{M}$ guanidine- $\mathrm{HCl}$. Purified protein was renatured slowly by dialysis against $\mathrm{H}$ buffer (20 mM HEPES [pH 7.9], 0.2 mM EDTA, $1 \mathrm{mM}$ dithiothreitol, $0.1 \%$ NP-40 $0.5 \mathrm{mM}$ phenylmethylsulfonyl fluoride) plus $300 \mathrm{mM} \mathrm{KCl}$ containing $3 \mathrm{M}, 1.5 \mathrm{M}, 1 \mathrm{M}, 0.5$ $\mathrm{M}$, and no guanidine- $\mathrm{HCl}$, respectively. For corenaturation experiments, proteins were diluted $1: 10$ in $6 \mathrm{M}$ guanidine$\mathrm{HCl}$ and then mixed at ratios of $1: 1,1: 5$, and $1: 20$ prior to dialysis.

For preparation of crude $E$. coli lysate containing p50, p65 $\Delta$, and p65 mutant proteins, mid-logarithmic-phase cultures were induced with $1 \mathrm{mM}$ IPTG for $2.5 \mathrm{~h}$, pelleted, and resuspended in $\mathrm{H}$ buffer without NP-40. Cells were lysed by sonication with two 30-s cycles, and lysates were cleared by centrifugation and frozen at $-70^{\circ} \mathrm{C}$.

Electrophoretic mobility shift assays. Binding reactions were carried out as previously described (46) with the addition of dithiothreitol $(1 \mathrm{mM})$ and NP-40 $(0.1 \%)$ to the binding buffer. Translation lysates $(2 \mu \mathrm{l})$ or bacterial protein ( $\sim 20 \mathrm{ng})$ and ${ }^{32} \mathrm{P}$-labeled probe $(1.5 \mathrm{ng} ; 500,000 \mathrm{cpm})$ were used in the binding reactions. Nondenaturing polyacrylamide gels $(4 \%)$ were electrophoresed at room temperature in a Tris-acetate buffer (6.7 $\mathrm{mM}$ Tris- $\mathrm{Cl}$ [pH 7.5], $3.3 \mathrm{mM}$ sodium acetate, $1 \mathrm{mM}$ EDTA) with recirculation for $2 \mathrm{~h}$ for binding reactions with translation lysate. Binding reactions with bacterial protein were run at $4^{\circ} \mathrm{C}$ in a Tris-borate buffer $(0.5 \times$ TBE) for $2 \mathrm{~h}$. For association experiments, electrophoresis was carried out for $3.5 \mathrm{~h}$. The $\kappa B$ probe used in the binding reactions contains the sequence 5'-GGATCCTCAA CAGAGGGGACTTTCCGAGGCCA-3', which corresponds to the $\kappa B$ motif present in the immunoglobulin light-chain enhancer.

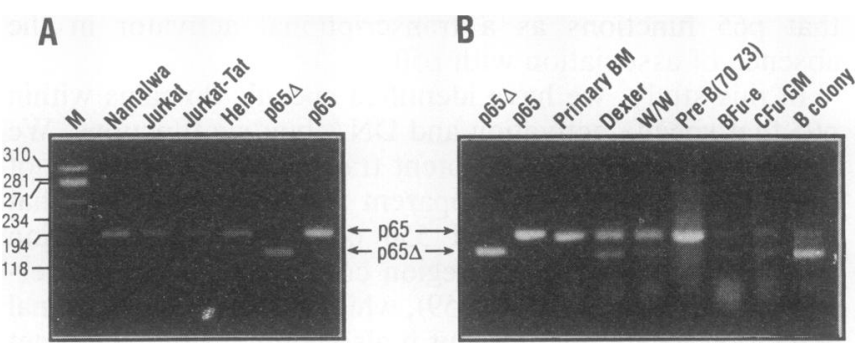

FIG. 1. PCR analysis of $\mathrm{p} 65$ and p65 transcription. (A) Analysis of PCR-amplified p65 and p65 RNAs from established human cell lines. Shown are PCR-amplified cDNA products from (left to right) the human B-lymphocyte line Namalwa, Jurkat $T$ lymphocytes, Jurkat T lymphocytes expressing Tat, and HeLa cells. (B) PCRamplified cDNA products from (left to right) murine primary bone marrow, bone marrow-derived colonies established according to Dexter et al. (11) and Whitlock and Witte (61), 70Z/3 (a pre-B cell line), bone marrow-derived erythroid (34) and macrophage (36) progenitors, and a B-cell colony (13). Lanes Dexter, W/W, and CFu-GM represent early myeloid progenitors, whereas lane BFu-e represents an early erythroid progenitor. The B-cell colony was obtained from the Whitlock-Witte lymphoid progenitors.

\section{RESULTS}

NF-kB p65 $\Delta$, a naturally occurring derivative of p65. The cDNAs encoding the murine (37) and human (45) NF-кB p65 proteins have recently been described. Upon sequencing six of our full-length p65 cDNA clones, isolated from a Jurkat T-cell library, it was observed that three clones lacked nucleotides 663 to 692 , encoding amino acids 222 to 231 (hereafter referred to as p65 $\Delta$ ). To establish the representation of this deletion in the mRNA population, the presence of p65 $\Delta$ RNA was examined by PCR analysis $(49,50)$ of cDNA prepared from various cell lines. The primer pairs chosen, which flank the deletion, were designed to yield a $180-\mathrm{bp}$ product for full-length mRNA species and a 150-bp product corresponding to the $30 \mathrm{bp}$ deleted in p65 $\Delta$. The results clearly show that both forms of p65 mRNA are produced (Fig. 1). In human cell lines thus far examined, the full-length species was most abundant (Fig. 1A). In contrast, several primary cell colonies derived from murine bone marrow demonstrated significant levels of p65 $\Delta$ expression (Fig. 1B). Although not readily evident from the PCR analysis of human RNA, the p65 $\Delta$ form must be present at significant levels, as $50 \%$ of the clones obtained from the Jurkat T-cell cDNA library, which was prepared with the same RNA sample as that used for the PCR, were representative of the p $65 \Delta$ form. This observation may suggest that p65 serves as a more efficient template for Taq polymerase, suggesting that the ratio of the p65 to p65 5 species may be somewhat skewed when the full-length p65 species is more prevalent. Consistent with this prediction, we have observed that PCR amplification of a sample containing an equal proportion of cloned p65 and p $65 \Delta$ cDNA favors amplification of the larger, p65 species.

The mechanism for generation of p65 $\Delta$ RNA was determined by examination of the intron-exon structure surrounding the deletion. Using the PCR primers described above, we amplified human genomic DNA and subcloned the amplified product. DNA sequencing of this region revealed that p65 $\Delta$ is produced via recognition of an alternate splice acceptor sequence present 30 nucleotides downstream of the authentic p65 splice acceptor (Fig. 2A). While the splice acceptor for p65 conforms to a consensus acceptor sequence, the 
A

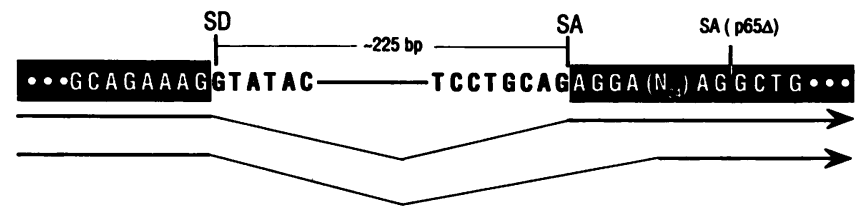

B

\begin{tabular}{|c|c|}
\hline $\begin{array}{l}\text { hNF - KB p65 } \\
\text { hNF - KB p65 } \\
\text { mNF- KB p65 } \\
\text { mEREL } \\
\text { heREL } \\
\text { mNF- KBp50 } \\
\text { DORSAL }\end{array}$ & 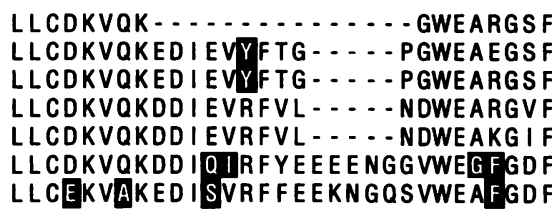 \\
\hline
\end{tabular}

FIG. 2. Schematic representation of the exon-intron structure encompassing the p65 alternative splice site. (A) Shaded regions correspond to exons. Splice donor and acceptor sites are marked SD and SA, respectively. (B) The coding sequence surrounding the alternate splice is aligned with sequences of mouse (22) (mcREL) and human (7) (hcREL) Rel and Rel-related gene products Drosophila dorsal (55) (DORSAL), mouse p50 (19) (mNF-kBp50), human p65 (45) (hNF-kBp65), and mouse p65 (37) (mNF-kBp65). The Rel homology domain of p65 encompasses approximately the first 300 amino acids of the 550-amino-acid protein, placing the alternate splice (removal of amino acids 222 to 231) at the $3^{\prime}$ portion of this domain. Following the Rel homology, the sequences diverge among each of the related family members. alternate acceptor for $\mathrm{p} 65 \Delta$ diverges from the consensus. Comparison of the amino acids encoded by the sequence encompassing and immediately adjacent to the splice demonstrates that it is well conserved among the various rel and rel-related genes, suggesting a functional significance for this region (Fig. 2B).

Transcriptional activity of p65. Earlier studies raised the possibility that the DNA binding domain and transcriptional activation functions of the NF- $\mathrm{kB}$ complex are distinct and would be conferred by the p50 and p65 subunits, respectively. However, recent reports showing that p65 can interact with $\kappa B$ DNA in the absence of $p 50(37,45,60)$ raised the possibility that p65 might confer both functions on its own.

To examine the functions of $\mathrm{p} 65$ and $\mathrm{p} 65 \Delta$, eukaryotic expression vectors were constructed and the ability of these proteins to stimulate transcription from various NF-kBresponsive CAT reporter plasmids was tested (Fig. 3). Jurkat $T$ lymphocytes were used in transfection experiments since they produce active NF- $\mathrm{kB}$ in response to mitogen stimulation $(5,33,44)$. The ability of the cells to support activation of the HкB-4 CAT reporter construct (33) was first demonstrated by confirming that expression of the human T-cell leukemia virus type I (HTLV-I) Tax protein $(14,15,54)$, a potent activator of $\mathrm{NF}-\mathrm{kB}(5,33,44)$, could stimulate transcription from HкB-4 (not shown). Plasmid HкB-4 contains four tandem copies of the $\mathrm{kB}$ motif present in the HIV long terminal repeat (LTR) (33). Upon cotransfection with CMV-p65, a dramatic stimulation of HкB-4 activity was also evident (Fig. 3A). No stimulation was obtained with cotransfection of CMV-p65 . Similar findings were obtained with plasmid ILR-kB-4 (33), which contains four tandem copies of the $\mathrm{\kappa B}$ motif present in the IL-2R $\alpha$ promoter (Fig. 3B). Immunoprecipitation analysis using epitope-tagged p65 and

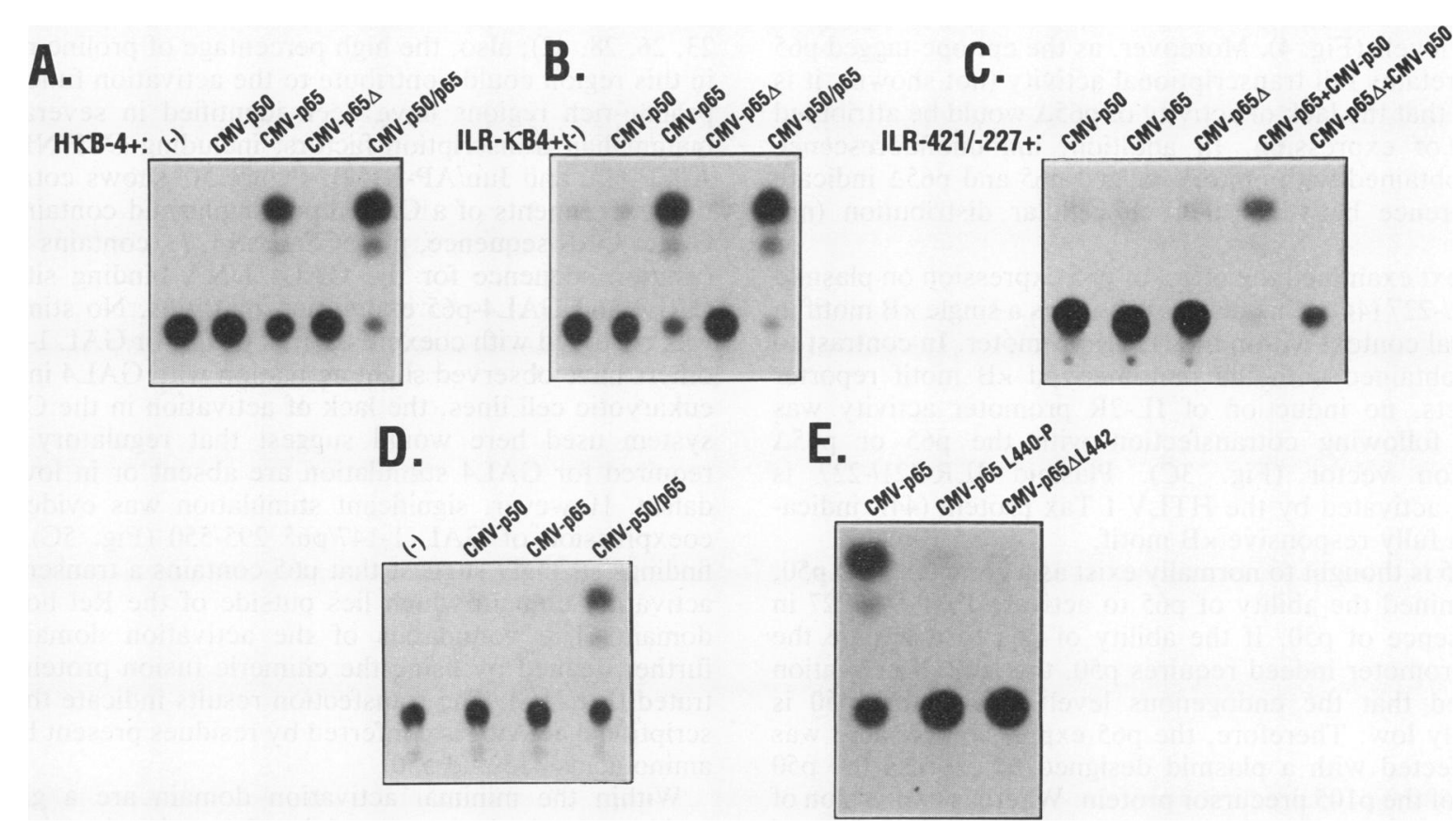

FIG. 3. Functional analysis of $p 50$ and $p 65$ in transient transfection assays. Jurkat cells were transfected with the reporter plasmid

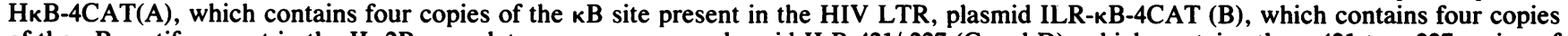
of the $\mathrm{kB}$ motif present in the IL-2R $\alpha$ regulatory sequence, or plasmid ILR-421/-227 (C and D), which contains the -421 to -227 region of the IL-2R enhancer. (E) CAT assays from Jurkat cells transfected with the HкB-4CAT reporter and the expression plasmid encoding the p65 mutations indicated. Transfections were performed in the absence (-) or presence of the expression plasmids indicated. Cells were harvested $48 \mathrm{~h}$ posttransfection, and CAT assays were performed. CMV-p50/p65 is a chimeric gene containing the sequences that encode amino acids 1 to 370 of p50 and 309 to 550 of p65. 


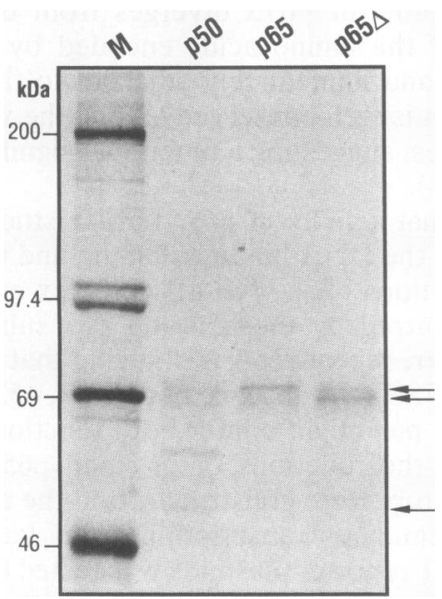

FIG. 4. Immunoprecipitation analysis of epitope-tagged p50, p65, and p $65 \Delta$ proteins. COS cells transfected with expression vectors carrying the $\mathrm{p} 65, \mathrm{p} 50$, and p $65 \Delta$ genes tagged with a 10-residue influenza virus HA epitope (30) at the amino terminus were labelled with $\left[{ }^{35} \mathrm{~S}\right]$ cysteine $48 \mathrm{~h}$ posttransfection. Tagged proteins were immunoprecipitated with anti-HA antibody (BAb Co) and analyzed on $10 \%$ polyacrylamide gels. Specific immunoprecipitated products are indicated by bars. The bands comigrating with the p65/65 proteins (upper arrow) represent background protein products that are sometimes precipitated by the HA serum and are observed in each lane. The lower arrow corresponds to the HAtagged $\mathrm{p} 50$ protein. The upper bands in the $\mathrm{p} 50$ lane are specific to the HA-p50 immunoprecipitations and may represent posttranslationally modified protein or Rel-related proteins that are precipitated via their association with p50.

p65 $\Delta$ proteins revealed that the two proteins were expressed at equal levels (Fig. 4). Moreover, as the epitope-tagged p65 protein retains full transcriptional activity (not shown), it is unlikely that the lack of activity of p65 $\Delta$ would be attributed to lack of expression. In addition, immunofluorescence results obtained with epitope-tagged p65 and p65 indicate no difference between their subcellular distribution (not shown).

We next examined the effect of p65 expression on plasmid ILR-421/-227 (44). This plasmid contains a single $\kappa B$ motif in its natural context within the IL-2R promoter. In contrast to results obtained with the multimerized $\kappa B$ motif reporter constructs, no induction of IL-2R promoter activity was evident following cotransfection with the p65 or p65 expression vector (Fig. 3C). Plasmid ILR-421/-227 is strongly activated by the HTLV-I Tax protein (44), indicative of a fully responsive $\kappa B$ motif.

As p65 is thought to normally exist as a complex with p50, we examined the ability of p65 to activate ILR-421/-227 in the presence of p50. If the ability of p65 to stimulate the IL-2R promoter indeed requires $\mathrm{p} 50$, the lack of activation suggested that the endogenous level of available p50 is extremely low. Therefore, the p65 expression plasmid was cotransfected with a plasmid designed to express the p50 portion of the p105 precursor protein. Whereas expression of p50 alone had no effect on induction, cotransfection with p65 led to a marked stimulation of CAT activity (Fig. 3C). Once again, no stimulation was evident with coexpression of p65 $\Delta$.

The findings presented above demonstrate that p65 can elicit stimulation of a multimeric $\mathrm{\kappa B}$ motif in the absence of p50 but requires association with p50 when the $\kappa B$ motif is present within certain natural contexts. This finding suggested that p50 may exhibit a stronger association to the natural $\kappa B$ motif than does $\mathrm{p} 65$, consistent with in vitro studies demonstrating that p65 has a 20 -fold-lower affinity than does $\mathrm{p} 50$ for the $\kappa \mathrm{B}$ site (60). To examine this possibility, a chimeric protein containing the DNA binding domain of p50 and carboxy-terminal activation domain of p65 (see below) was constructed. This $\mathrm{p} 50 / \mathrm{p} 65$ fusion protein induced activation from both the multimeric and natural-context $\kappa B$ motifs (Fig. 3D), in agreement with the findings presented above. The ability of the p50/p65 chimera to activate transcription provides further support for the presence of an activation domain within the carboxy terminus of p65.

Localization of the p65 activation domain. Transfected p65 may stimulate transcription directly by association with the transcriptional machinery or by association with preexisting cellular p50. However, it is unlikely that the effects observed reflect association of p65 with cellular p50, since expression of p65 was unable to induce activation of the ILR-421/-227 reporter in the absence of cotransfected p50. Nevertheless, both of these mechanisms suggest that p65 $\Delta$ lacks a functional activation domain or is unable to associate with either itself or p50. Alternatively, the activation obtained upon cotransfection with CMV-p65 may be an indirect effect reflecting the ability of excess p65 to interact with the NF-кB cytosolic inhibitor, IкB, thus allowing translocation of preexisting p50-p65 complexes into the nucleus. This would argue that $p 65 \Delta$ is unable to interact with IкB.

To distinguish between these possibilities, the presence and location of the p65 activator domain were identified by construction of a chimeric protein containing amino acids 1 to 147 (DNA binding domain) of the yeast transcriptional activator GAL4 and amino acids 295 to 550 of p65 (Fig. 5). We focused on the carboxy terminus, as this region in both Rel and dorsal contributes to the activation function $(8,16$, $23,26,28,42$ ); also, the high percentage of proline residues in this region could contribute to the activation function, as proline-rich regions have been identified in several other mammalian transcription factors, including CTF/NF1 (35), AP-2 (62), and Jun/AP-1 (57). Figure 5C shows cotransfection experiments of a CAT reporter plasmid containing the GAL4 UAS sequence, pGMCS/UAS (27) (contains the recognition sequence for the GAL4 DNA binding site) with GAL4 and GAL4-p65 expression plasmids. No stimulation was observed with coexpression of GAL4 or GAL 1-147. As others have observed slight activation with GAL4 in certain eukaryotic cell lines, the lack of activation in the COS cell system used here would suggest that regulatory factors required for GAL4 stimulation are absent or in low abundance. However, significant stimulation was evident with coexpression of GAL 1-147/p65 295-550 (Fig. 5C). These findings strongly suggest that p65 contains a transcriptional activation domain which lies outside of the Rel homology domain. The boundaries of the activation domain were further defined by using the chimeric fusion proteins illustrated (Fig. 5C). The transfection results indicate that transcriptional activity is conferred by residues present between amino acids 415 and 550 .

Within the minimal activation domain are a group of residues that have the potential to form a leucine zipper-like motif (Fig. 5B) (31). The importance of this region for p65 function was examined by creating mutations GAL/L440-P and GAL/ $\Delta \mathrm{L} 442$, which replace leucine 440 with a proline and delete leucine 442 , respectively. These mutations were made within the GAL4/p65 295-550 vector. Insertion of a proline would be predicted to partially disrupt the alpha- 


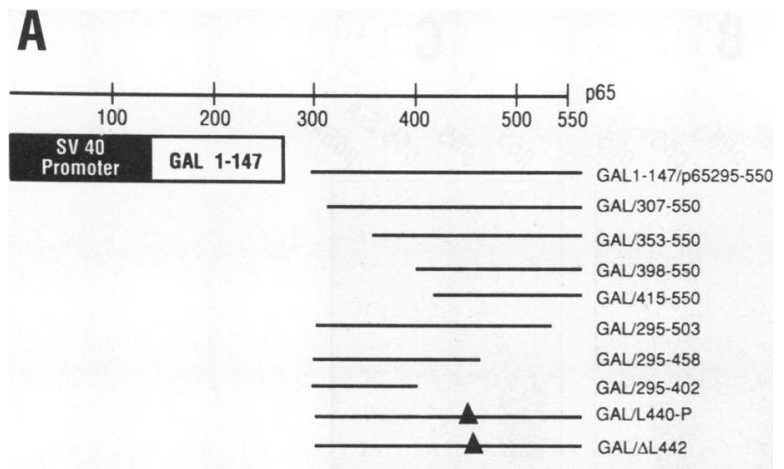

GTLSEALLQ LQ FDDEDLGA

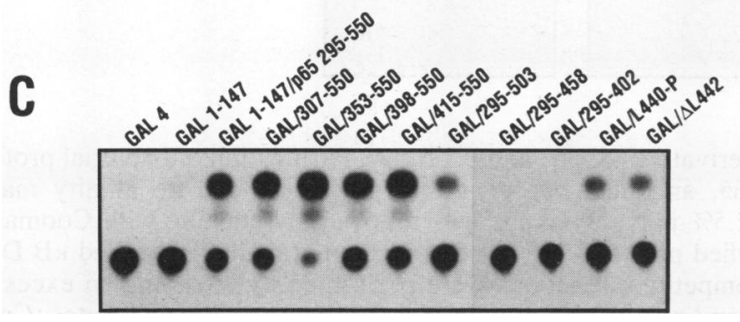

FIG. 5. Mapping of the p65 activation domain. (A) Schematic drawing of the GAL4-p65 chimeric proteins driven by the simian virus 40 (SV40) promoter. The indicated region of p65 was amplified by PCR and cloned in frame with the DNA binding domain of GAL4. The amino acids of p65 present within the chimeric molecule are indicated within the name of the construct. Mutations in the putative leucine zipper motif were made in the GAL4 1-147/p65 295-550 protein. (B) Residues present in the potential leucine zipper motif. (C) Transient cotransfection of chimeric GAL4/p65 proteins into Cos 7 cells. The plasmid DNAs shown in panel A were cotransfected into Cos 7 cells together with a CAT reporter plasmid containing the GAL4-responsive UAS sequence, upstream of the mouse mammary tumor virus promoter (plasmid pGMCS $\triangle$ GRE/ UAS) lacking the glucocorticoid response element. Plasmid pAG4 encodes a full-length GAL4 protein, and pSG424 encodes amino acids 1 to 147 specifying the GAL4 DNA binding domain. Cells were harvested $48 \mathrm{~h}$ posttransfection, and CAT assays were performed.

helical nature of this region. Both fusion proteins exhibited reduced activity with respect to the parental construct, thus establishing the importance of this domain for activation function in the context of the GAL4-p65 fusion protein (Fig. 6C). However, the importance of this region for transcriptional activity was further demonstrated by showing that the chimeric construct GAL/295-458, which deletes from the $\mathrm{COOH}$ terminus to amino acid 458, removing residues predicted to contribute to the alpha-helical nature of this region, was no longer active. We also note that the presence of additional residues likely contributes to the activation function, at least in the context of the GAL4 chimeras, as residues between 503 and 550 appear to be required for maximal transcription induction (i.e., compare the activities of plasmids GAL/295-550 and GAL/295-503).

Having established the importance of residues 435 to 459 for activation in the context of the GAL4-p65 chimeric protein, the role of these residues for function in authentic p65 was examined. Two site-directed mutations, producing changes identical to those produced in plasmids GAL/ L440-P and GAL/ $\triangle$ L442 (i.e., a change of leucine residue 440 to proline and deletion of leucine residue 442 , respectively) were made within plasmid CMV-p65, and the tran- scriptional activity of the expressed protein was assessed in a cotransfection assay with the $\mathrm{H \kappa B}-4 \mathrm{CAT}$ reporter plasmid (Fig. 3E). These mutations had a more dramatic effect than that observed with similar disruptions in the GAL4/p65 chimeras in that no measurable transcriptional activity was observed with the кB CAT reporter used. Taken together, the two sets of results indicate that residues 435 to 459 are essential for the p65 transcriptional activation function.

Identification of a domain essential for interaction with $\mathbf{~} B$ DNA. The results reported thus far demonstrate that p65 is a potent transcriptional activator and that its activation domain resides outside of the region affected by the p65 deletion. Therefore, the inability of p $65 \Delta$ to function in either the absence or presence of p50 would be fully consistent with a defect in DNA binding or multimerization. To examine this possibility, DNA binding was examined by testing the ability of bacterially synthesized p65, p 50 , and p65 truncated proteins, containing those residues shown previously to be sufficient for DNA interaction $(19,29,37)$, to associate with each other and with $\mathrm{kB}$ DNA. Purification of each protein was achieved by addition of a six-histidine residue moiety at the amino terminus, which facilitates purification using a nickel chelate affinity resin (17). With use of either purified p 65 or p 50 or crude $E$. coli lysates containing these proteins, strong association with $\mathrm{\kappa B}$ DNA was readily obtained (Fig. 6). No association was evident with p65 5 protein. The importance of the domain corresponding to the p65 deletion for DNA binding was further established by showing that protein $\mathrm{p} 65 \mathrm{~K} / \mathrm{I}$, containing a point mutation changing lysine residue 221 to isoleucine, or protein p65E/A, changing glutamic acid residue 225 to alanine, could no longer associate with DNA (Fig. 6C).

The ability of p 65 and p $65 \Delta$ to associate with p 50 was next examined. Because of the strong association of the p50 subunits in the homodimer, association was measured by renaturing p50 together with increasing amounts of p65 or p $65 \Delta$, followed by analysis of DNA binding complexes (Fig. 7). p65 readily formed a complex with p50, as suggested by the presence of an intermediate-size complex between p65 and p50 homomeric $\mathrm{\kappa B}$ DNA-protein complexes. No intermediate-size complexes were observed between p50 and p $65 \Delta$. However, incubation of p65 with a 20 -fold molar excess of p65 did reduce the ability of p65 to interact with DNA, suggesting that p65 can associate with $\mathrm{p} 65$.

The foregoing results obtained by using the gel mobility shift analysis suggest that residues 222 to 231 , absent in p $65 \Delta$, contribute to multimerization. A coimmunoprecipitation assay was used to examine by a more direct means the ability of these proteins to associate with one another. As each of the antibodies in our possession recognized the Rel homology domain, they were nondiscriminatory against the individual proteins. Similarly, the fact that these antibodies may recognize determinants within the putative multimerization domain, the antibody-protein association could possibly interfere with associations between the individual Rel-related proteins. To avoid these potential problems, the following approach was taken. Briefly, the cDNA corresponding to either the full-length or truncated $\mathrm{p} 50$, p65, or p65 protein was cloned into an in vitro transcription vector in frame with an oligonucleotide encoding nine residues corresponding to a domain present in the influenza virus HA protein (30). RNA corresponding to the epitope-tagged protein was transcribed in vitro by using T7 RNA polymerase and was used in combination with RNA coding for nontagged protein to program an in vitro rabbit reticulocyte translation lysate. As would be predicted, HA-tagged p50 


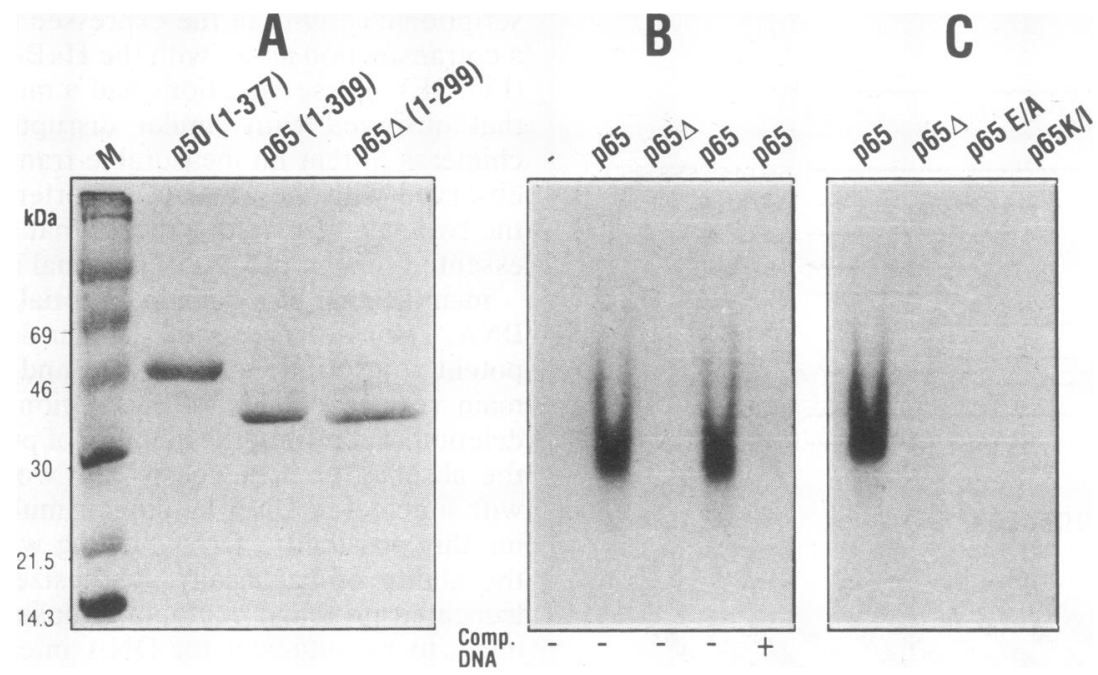

FIG. 6. Analysis of DNA binding activity of bacterial purified truncated derivatives of p65 and p65 . (A) Purification of bacterial protein. The histidine-tagged truncated protein products corresponding to p 50 , p65, and p65 $\Delta$ were purified by nickel chelate affinity matrix chromatography as described in Materials and Methods and analyzed on $12.5 \%$ polyacrylamide gels followed by staining with Coomassie blue. (B) DNA binding activity of purified p65 (1-309) and p65 (1-299). Purified protein ( $\sim 20 \mathrm{ng})$ was incubated with ${ }^{32}$ P-labelled kB DNA and binding buffer and then analyzed on $4 \%$ native polyacrylamide gels. Competition reactions were performed by including an excess of unlabelled $\mathrm{\kappa B}$ DNA in the binding reaction. (C) DNA binding activity of p65 and mutant p65 proteins present in crude $E$. coli lysates. Crude lysates from induced cultures of $E$. coli expressing the p65 mutant proteins indicated were prepared by sonication (see Materials and Methods), and $2.0 \mu \mathrm{l}$ of extract was incubated with ${ }^{32} \mathrm{P}$-labelled $\mathrm{\kappa B}$ probe and analyzed on $4 \%$ native polyacrylamide gels.

coimmunoprecipitated p65 (Fig. 8). No association with p65 $\Delta$ was evident. In contrast, HA-tagged p65(1-309) was able to associate with $\mathrm{p} 65 \Delta$ in addition to being able to coimmunoprecipitate p50 (data not shown). No evidence for homodimer formation was obtained when HA-tagged p65 (1-309) was incubated with full-length p65 . These findings, together with the findings obtained from the gel mobility shift analysis, suggest that residues 222 to 231 contribute to multimerization and have a more pronounced effect on the p65 4 -p 50 association than on the p65-p65 association.

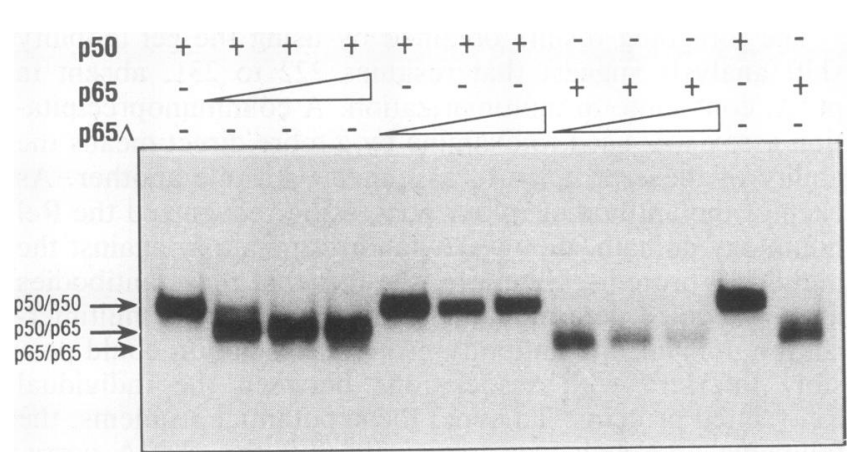

FIG. 7. Association of NF-kB truncated proteins as measured by gel mobility shift analysis. The purified proteins shown in Fig. 6A were mixed as shown and renatured as described in Materials and Methods. Renatured protein was incubated with ${ }^{32} \mathrm{P}$-labelled $\mathrm{KB}$ DNA in binding buffer and analyzed on $4 \%$ native polyacrylamide gels. The arrows indicate homomeric $\mathrm{p} 50$ and $\mathrm{p} 65$ complexes and the heteromeric p50/p65 complex. The triangles indicate increasing amounts of the respective protein (1-, 5-, and 20-fold molar excess) to a fixed amount $(10 \mathrm{ng})$ of the indicated protein with which it was renatured.
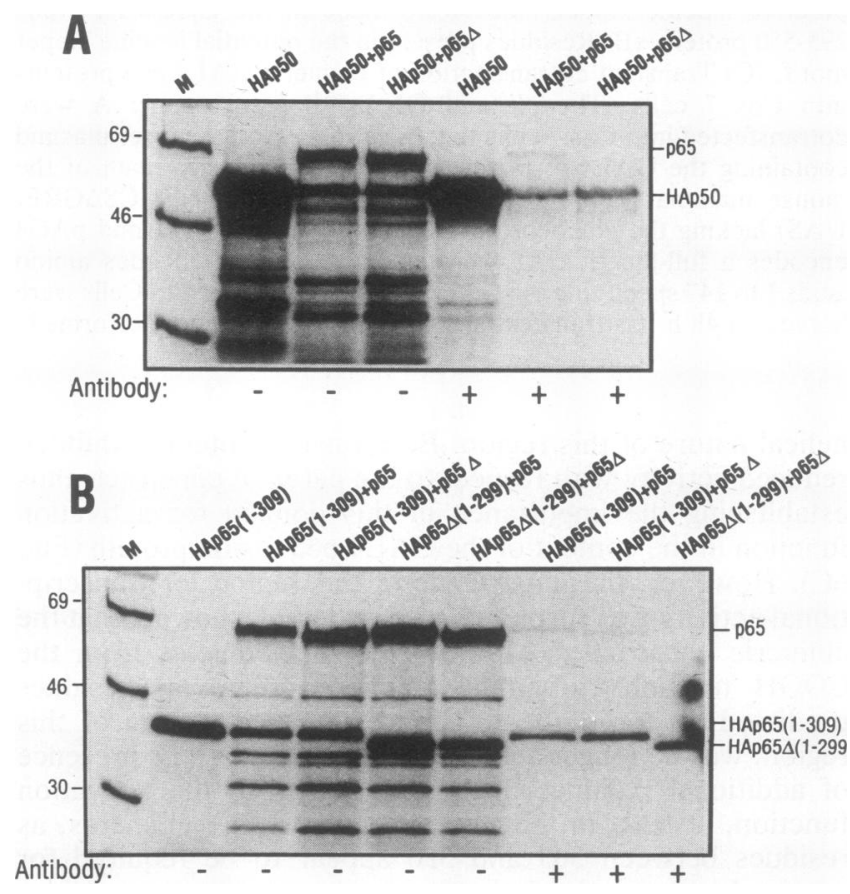

FIG. 8. Coimmunoprecipitation analysis of $\mathrm{p} 65$, p $65 \Delta$, and $\mathrm{p} 50$ associations. The in vitro-transcribed RNAs corresponding to the protein products shown were cotranslated in a rabbit reticulocyte translation lysate. The lanes depict translation reactions prior to immunoprecipitation $(-)$ or products obtained following immunoprecipitation with the anti-HA serum $(+)$. Translation reactions shown in panel A all contained HA-tagged p50 protein together with either nontagged p65 or p65 4 . In panel B, reactions contained either HA-tagged p65 (1-309) or p65 (1-299) with nontagged p50, fulllength p65, or p65 . 


\section{DISCUSSION}

We used a naturally occurring, alternatively spliced variant of NF-kB p65 to help define functional domains present in p65. Using vectors that overexpress either the full-length or truncated form of p65, we observed that $\mathrm{p} 65$ is a potent transcriptional activator of reporter constructs bearing a $\mathrm{kB}$ recognition motif, whereas p65 $\Delta$ and p50 are not. This finding is fully consistent with previous reports suggesting that p 50 confers DNA binding specificity and p65 confers an activation function for the NF-kB motif $(29,59)$. However, in light of recent studies demonstrating the ability of fulllength or truncated p65 to associate with $\kappa B$ DNA in the absence of p50 $(37,45,60)$, the function of p 65 in the absence of exogenously added p50 was examined. p65 was able to activate transcription of reporter plasmids bearing multimeric copies of the $\kappa B$ motif derived from either the HIV LTR or IL-2R promoter. Surprisingly, no activation was evident with the reporter containing the $\kappa B$ motif present in its natural context within the IL-2R regulatory region. The discordance between the activation function on a multimeric versus a natural-context $\mathrm{\kappa B}$ motif can be interpreted in several ways. First, p65 might interact with certain $\kappa B$ elements on its own and may require association of p50 for interaction with others. This may represent a threshold effect, suggesting that a critical number of p65 molecules may need to bind in order to effect transcription. This would be consistent with the different affinities of p65 and p50 for the $\kappa B$ motif $(60)$. Our observation that the IL-2R $\kappa B$ motif could indeed be activated when p65 is expressed with p50 supports this model. The inability to obtain activation of the IL-2 $R$ promoter in the absence of cotransfection with the p50 expression plasmid also indicates that the endogenous level of p50 must be extremely low, providing further support that activation of the tandem $\kappa \mathrm{B}$ motifs by p65 likely occurs in the absence of p50. In agreement with this suggestion, Northern (RNA) blot analysis of Jurkat T-cell RNA obtained from nonstimulated cells failed to detect a significant amount of p50 mRNA (unpublished observations). The ability of a fusion protein containing the DNA binding domain of p50 and activation domain of p65 (see below) to induce activity with each of the $\kappa \mathrm{B}$ motifs further suggests that the higher affinity of p50 for $\kappa B$ DNA allows it to bring p65 to a wider variety of naturally occurring $\kappa B$ sites in different structural contexts. Nonetheless, the ability of p65 to activate transcription, in the apparent absence of p50, raises the intriguing possibility that NF- $\mathrm{KB}$ function will not always require the presence of the $\mathrm{p} 50$ subunit. The ability of p65 to activate the HIV LTR (not shown), which contains two copies of the $\mathrm{\kappa B}$ motif, in the apparent absence of p50 would be consistent with this prediction. Alternatively, the ability of p65 to function in the absence of p50 with the multimerized $\kappa B$ motif may reflect a cooperative effect of the four tandem binding sites that may not occur in nature. Therefore, the ability of p65 to function in the absolute absence of p50 on a naturally occurring $\mathrm{\kappa B}$ motif remains to be established.

The inability of p $65 \Delta$ to induce transcriptional activation raised the possibility that amino acids 222 to 231 contribute to an essential binding or multimerization function or reside within the activation domain. To address the latter possibility, the ability of chimeric fusion proteins containing the DNA binding domain of the yeast transcriptional activator GAL4 and the carboxy-terminal domain of p65 to activate a reporter plasmid containing the GAL4 UAS was examined. The ability of several of the fusion proteins to activate the
GAL4 UAS is consistent with the premise of an activation domain within the carboxy terminus of p65. This finding is also consistent with earlier reports demonstrating the presence of an activation domain in the carboxy terminus of Rel $(8,16,23,28,42)$ and the carboxy terminus of dorsal (26). Because p50 lacks transcriptional activity, the strong induction obtained with the p50/p65 chimeric protein is also fully consistent with this result. Deletion analysis of the activation domain mapped a minimal activation domain to 135 amino acids between residues 415 to 550 of the carboxy terminus.

It is intriguing that slight alterations within residues 435 to 459 , a region that bears resemblance to a leucine zipper-like motif, drastically reduces the activity of the chimeric proteins. The loss of activity experienced with plasmid GAL/ p65 295-458, which lacks the $3^{\prime}$ portion of this sequence and is predicted to contribute to the alpha-helical structure of this proposed domain, further supports the importance of this region for transcriptional activation functions. As this region has an overall net negative charge, a feature common to other activation domains, the precise nature of those determinants that confer the activation function remains to be established. However, mutation of leucine residue 440 to proline, which would be predicted to disrupt the alphahelical structure, and deletion of leucine residue 442 , which disrupts the spacing between leucines in authentic p65, abolished transcriptional activity. This finding suggests that these parameters within this region are necessary for the complete activation function. The prototypic leucine zipper originally identified by Landschulz and colleagues (31) consists of a heptad repeat of leucine residues in which leucine residues are repeated at intervals of seven residues. It is thought that the two alpha helices interact in a parallel orientation to form a structure reminiscent of a coiled-coil structure originally identified in keratin and myosin protein families (39). Leucine zippers have since been identified in many transcriptional regulatory proteins $(9,63)$. The residues present in p65 do not conform to the prototype configuration, wherein the leucine zipper contains a positively charged region immediately adjacent to the amino terminus of the zipper domain. However, in p65, residues 435 to 459 do exhibit a high degree of charged amino acids ( 5 of 15 ) and would be predicted to form an alpha-helical structure, a feature common in leucine zipper motifs. If indeed the p65 activation domain is authentic, our findings suggest that the ability of p65 to activate transcription through association with other regulatory factors might occur through a leucine zipper-type interaction.

As the p65 activation function is far removed from the region deleted from p $65 \Delta$, the importance of deleted residues 222 to 231 for DNA binding was examined. Using bacterially expressed p65 and p65 5 proteins in a gel mobility shift analysis, we observed that p65 readily associates with p50, in accord with previous findings, whereas p65 does not. Furthermore, the importance of residues corresponding to the p $65 \Delta$ deletion was clearly established, as evidenced by the inability of proteins $\mathrm{p} 65 \mathrm{~K} / \mathrm{I}$ and $\mathrm{p} 65 \mathrm{E} / \mathrm{A}$, which contain single amino acid changes (lysine residue 221 to isoleucine and glutamic acid residue 225 to alanine, respectively), to bind $\kappa B$ DNA.

The inability of p65 to associate with DNA can be interpreted in several ways. For example, the defect in p65 could reflect a deletion of a DNA binding motif. Alternatively, as DNA binding likely requires multimerization of p65 with itself or p50 $(3,59)$, a defect in the multimerization domain would also provide a negative result in a gel mobility 
shift assay. If the defect in p65 $\Delta$ reflects disruption of the DNA binding motif, then mixed multimers of p65 2 with p65 or p50 would most likely be unable to interact with DNA. In corenaturation/mixing experiments of $\mathrm{p} 65 \Delta$ with $\mathrm{p} 50$, no evidence for protein association or lessening of p50 DNA binding was observed. Similarly, no evidence for association of p65 with p 50 was obtained in the coimmunoprecipitation analysis. In contrast, renaturation of p65 with increasing amounts of p $65 \Delta$ reduced the ability of p65 to bind $\kappa B$ DNA. Consistent with this finding were results obtained from the coimmunoprecipitation analysis showing that $\mathrm{p} 65 \Delta$ can associate with $\mathrm{p} 65$, although no evidence for homodimer formation was obtained. These findings would suggest that amino acids 222 to 231 are i equired for association of p65 with p50. Moreover, the ability of p65 to interact with p65 and not with itself suggests that the residues remaining in p65 may contribute a minimal multimerization activity that is sufficient for function when presented with an intact multimerization domain, as is the case with p65. Thus, the inability of $\mathrm{p} 65 \Delta$ to associate with $\mathrm{p} 50$, which would also contribute an intact multimerization domain, could easily reflect subtle differences between those residues that specify the multimerization functions of these proteins. Our data do not address the nature of the multimer formation, nor do they rule out a contribution of other residues in this process. Furthermore, our findings cannot address whether the p65p65 heteromeric complex interacts with DNA. For example, the ability of p $65 \Delta$ to reduce the ability of p65 to interact with $\mathrm{\kappa B}$ DNA could reflect the formation of heteromeric complexes that have greatly reduced DNA binding capabilities. If this is true, these findings raise the possibility that those residues which contribute to multimerization lie close to, or coincide with, those residues that contribute to DNA binding.

Our findings suggesting a role of amino acids 222 to 231 in multimerization do not address whether this region is also important for interaction with the cytosolic repressor IkB. These residues may not be required for conferring direct association but may be indirectly required, in that ІкB may recognize the p65 dimer or p65-p50 complex. Therefore, if these residues indeed contribute to the ability of p65 to associate with $\mathrm{I} \kappa \mathrm{B}$, the transcription induction experienced with p65 could be an indirect rather than direct effect, resulting from removal of IKB from preexisting p65-p50 complexes. However, we feel that this is unlikely, as the inability of p65 to induce activation of the IL-2R promoter was reversed by coexpression of $\mathrm{p} 50$. The importance of residues 222 to 231 for IKB interaction will undoubtedly be answered, as IкB has recently been cloned (24).

Finally, the physiological role of $\mathrm{p} 65 \Delta$ in the NF-kB transcription pathway remains to be established. Although p65 lacks the ability to stimulate transcription through the NF- $\kappa B$ motif, the relative abundance of this protein in murine cell lines at various stages of hematopoietic development suggests a physiological role for this protein in regulation or differentiation. Consistent with this suggestion, we have observed that overexpression of p $65 \Delta$ can elicit profound phenotypic differences in certain cell types (35a). One possibility awaiting further study is that $p 65 \Delta$ functions as a negative regulator of NF- $\mathrm{kB}$ function through formation of inactive heteromeric complexes with p65 or complexes with greatly reduced DNA binding capabilities. This presents the intriguing possibility that cell-specific differential splicing and expression of p $65 \Delta$ may alter expression of a set of genes that are regulated by NF-kB. Alternatively, if p $65 \Delta$ retains its ability to associate with the cytosolic repressor $\mathrm{I} \mathrm{KB}$, it may function as a regulatory "sink," soaking up excess IKB and thus allowing $\mathrm{p} 65$ or $\mathrm{p} 65 / \mathrm{p} 50$ complexes to enter the nucleus.

\section{ACKNOWLEDGMENTS}

We thank G. Nabel for plasmids HкB-4 CAT and ILR-кB-4 M. Ptashne for plasmids pGMCS/UAS and GAL4/1-147, W. Benjamin for support with the bone marrow colonies, P. Baeuerle and P. Dillon for helpful discussion, and T. Rose for preparation of the manuscript.

S. Ruben was the recipient of a fellowship from the Leukemia Society of America.

\section{REFERENCES}

1. Baeuerle, P. A., and D. Baltimore. 1988. IkB: a specific inhibitor of the NFKB transcription factor. Science 242:540-546.

2. Baeuerle, P. A., and D. Baltimore. 1988. Activation of DNAbinding activity in an apparently cytoplasmic precursor of the NFK B transcription factor. Cell 53:211-217.

3. Baeuerle, P. A., and D. Baltimore. 1989. A $65 \mathrm{kD}$ subunit of active NF-kB is required for inhibition of NF-kB. Genes Dev 3:1689-1698.

4. Baeuerle, P. A., and D. Baltimore. 1991. The physiology of the NF-kappa-B transcription factor. Mol. Aspects Cell. Regul. 6:409-432.

5. Ballard, D. W., E. Böhnlein, J. W. Lowenthal, Y. Wano, B. R. Franza, and W. C. Greene. 1988. HTLV-1 Tax induces cellular proteins that activate the $\mathrm{\kappa B}$ element in the IL-2 receptor alpha gene. Science 241:1652-1654.

6. Bohnlein, E., J. W. Loiwenthal, M. Siekevitz, D. W. Ballard, B. R. Franza, and W. C. Greene. 1988. The same inducible nuclear protein(s) regulates mitogen activation of both the interleukin-2 receptor-alpha gene and type 1 HIV. Cell 53:827836.

7. Brownell, E., N. Mittereder, and N. R. Rice. 1989. A human rel proto-oncogene cDNA containing an Alu fragment as a potential coding exon. Oncogene 4:935-942.

8. Bull, P., K. L. Morley, M. F. Hoekstra, T. Hunter, and I. M. Verma. 1990. The mouse c-rel protein has an $\mathrm{N}$-terminal regulatory domain and a C-terminal transcriptional transactivation domain. Mol. Cell. Biol. 10:5473-5485.

9. Busch, S. J., and P. Sassone-Corsi. 1990. Dimers, leucine zippers and DNA binding domains. Trends Genet. 6:36-40.

10. Capobianco, A. J., D. L. Simmons, and T. D. Gilmore. 1990 Cloning and expression of a chicken c-rel cDNA: unlike p59 (v-rel), p68 (c-rel) is a cytoplasmic protein in embryo fibroblasts. Oncogene 5:257-266.

11. Dexter, T. M., T. D. Allen, and L. G. Lajtha. 1977. Conditions controlling the proliferation of haemopoietic stem cells in vitro. J. Cell. Physiol. 91:335.

12. Dillon, P. J., P. Nelbock, A. Perkins, and C. A. Rosen. 1990 Function of the human immunodeficiency virus types 1 and 2 Rev proteins is dependent upon their ability to interact with a structured region present in the env gene mRNA. J. Virol. 64:4428-4437.

13. Dorshkind, K. 1990. Regulation of hemopoiesis by bone marrow stromal cells and their products. Annu. Rev. Immunol. 8:111137.

14. Felber, B. K., H. Paskalis, C. Kleinman-Ewing, F. Wong-Staal, and G. N. Pavlakis. 1985. The pX protein of HTLV-1 is a transcriptional activator of its long terminal repeat. Science 229:675-679.

15. Fujisawa, J., M. Seiki, T. Kiyokawa, and M. Yoshida. 1986 Functional activation of the long terminal repeat of human T-cell leukemia virus type I by a trans-acting factor. Proc. Natl. Acad. Sci. USA 82:2277-2281.

16. Gelinas, C., and H. M. Temin. 1988. The v-rel oncogene encodes a cell-specific transcriptional activator of certain pro- 
moters. Oncogene 3:349-355.

17. Gentz, R., C. H. Chen, and C. A. Rosen. 1989. Bioassay for trans-activation using purified human immunodeficiency virus tat-encoded protein: trans-activation requires mRNA synthesis. Proc. Natl. Acad. Sci. USA 86:821-824.

18. Ghosh, S., and D. Baltimore. 1990. Activation in vitro of NF-kB by phosphorylation of its inhibitor I-kappa-B. Nature (London) 344:678-682.

19. Ghosh, S., A. M. Gifford, L. B. Riviere, P. Tempst, G. P. Nolan, and D. Baltimore. 1990. Cloning of the p50 DNA binding subunit of NF-kB: homology to rel and dorsal. Cell 62:10191029.

20. Gilmore, T. D., and H. M. Temin. 1986. Different localization of the product of the $\mathrm{v}$-rel oncogene in chicken fibroblasts and spleen cells correlates with transformation by REV-T. Cell 44:791-800.

21. Gorman, C. M., L. F. Moffat, and B. H. Howard. 1982. Recombinant genomes which express chloramphenicol acetyltransferase in mammalian cells. Mol. Cell. Biol. 2:1044-1051.

22. Grumont, R. J., and S. Gerondakis. 1989. Structure of a mammalian c-rel protein deduced from the nucleotide sequence of murine cDNA clones. Oncogene 4:1-8.

23. Hannink, M., and H. M. Temin. 1989. Transactivation of gene expression by nuclear and cytoplasmic rel proteins. Mol. Cell. Biol. 9:4323-4336.

24. Haskill, S., A. A. Beg, S. M. Tompkins, J. S. Morris, A. D. Yurochko, A. Sampson-Johannes, K. Mondal, P. Ralph, and A. S. Baldwin, Jr. 1991. Characterization of an immediate-early gene induced in adherent monocytes that encodes IKB-like activity. Cell 65:1281-1289.

25. Ho, S. N., H. D. Hunt, R. M. Horton, J. K. Pullen, and L. R. Pease. 1989. Site-directed mutagenesis by overlap extension using the polymerase chain reaction. Gene 77:51-59.

26. Ip, Y. T., R. Kraut, M. Levine, and C. Rushlow. 1991. The dorsal morphogen is a sequence-specific DNA-binding protein that interacts with a long-range repression element in drosophila. Cell 64:439-446.

27. Kakidani, H., and M. Patshne. 1988. GAL4 activates gene expression in mammalian cells. Cell 52:161-167.

28. Kamens, J., P. Richardson, G. Mosialos, R. Brent, and T. Gilmore. 1990. Oncogenic transformation by v-Rel requires an amino-terminal activation domain. Mol. Cell. Biol. 10:28402847.

29. Kieran, M., V. Blank, F. Logeat, J. Vandekerchove, F. Lottspeich, O. LeBail, M. B. Urban, P. Kourilsky, P. A. Baeuerle, and A. Israel. 1990. The DNA binding subunit of NF-kB is identical to factor KBF1 and homologous to the rel oncogene product. Cell 62:1007-1018.

30. Kolodziej, P. A., and R. A. Young. 1991. Epitope tagging and protein surveillance. Methods Enzymol. 194:508-519.

31. Landschulz, W. H., P. F. Johnson, and S. L. McKnight. 1988. The leucine binding zipper: a hypothetical structure common to a new class of DNA binding proteins. Science 240:1759-1764.

32. Lenardo, M. J., and D. Baltimore. 1989. NF-kB: a pleiotropic mediator of inducible and tissue-specific gene control. Cell 58:227-229.

33. Leung, K., and G. Nabel. 1988. HTLV-1 transactivator induces interleukin-2 receptor expression through an NF-kB-like factor. Nature 333:776-778.

34. McLeod, D. L., M. M. Shreeve, and A. A. Axelrod. 1974. Improved plasma culture system for production of erythrocytic colonies in vitro: quantitative assay method of CFU-E. Blood 44:517.

35. Mermod, N., E. A. O'Neil, T. J. Kelly, and R. Tijan. 1989. The proline-rich transcriptional activator of CTF/NF-1 distinct from the replication and DNA binding domain. Cell 58:741.

35a.Narayanan, R., and C. A. Rosen. Unpublished data.

36. Narayanan, R., N. S. Tare, W. R. Benjamin, and U. Gubler. 1989. A sensitive technique to monitor gene transfer and expression in bone marrow stem cells. Exp. Hematol. 17:832835.

37. Nolan, G. P., S. Ghosh, H.-C. Liou, P. Tempst, and D. Baltimore. 1991. DNA binding and IKB inhibition of the cloned p65 subunit of NF-kB, a rel related polypeptide. Cell 64:961-969.

38. Osborn, L., S. Kunkel, and G. J. Nabel. 1989. Tumor necrosis factor alpha and interleukin-1 stimulate the human immunodeficiency virus enhancer by activation of the nuclear factor $\mathrm{kB}$. Proc. Natl. Acad. Sci. USA 86:2336-2340.

39. O'Shea, E. K., R. Rutkowski, and P. S. Kim. 1989. Evidence that the leucine zipper is a coiled coil. Science 243:538-542.

40. Queen, C., and D. Baltimore. 1983. Immunoglobulin gene transcription is activated by downstream sequence elements. Cell 33:741-748.

41. Rice, N. R., and R. V. Gilden. 1988. The rel oncogene, p. 495-512. In E. P. Reddy et al. (ed.), The oncogene handbook. Elsevier, New York.

42. Richardson, P., and T. Gilmore. 1991. v-Rel is an inactive member of the Rel family of transcriptional activating proteins. J. Virol. 65:3122-3130.

43. Roth, S., D. Stein, and C. Nusslein-Volhard. 1989. A gradient of nuclear localization of the dorsal protein determines dorsoventral pattern in the drosophila embryo. Cell 59:1189-1202.

44. Ruben, S., H. Poteat, T. H. Tan, K. Kawakami, R. Roeder, W. Haseltine, and C. A. Rosen. 1988. Cellular transcription factors and regulation of IL-2 receptor gene expression by HTLV-1 tax gene product. Science 241:89-91.

45. Ruben, S. M., P. J. Dillon, R. Schreck, T. Henkel, C.-H. Chen, M. Maher, P. A. Baeuerle, and C. A. Rosen. 1991. Isolation of a rel-related human cDNA that potentially encodes the $65-\mathrm{kD}$ subunit of NF-kB. Science 251:1490-1493.

46. Ruben, S. M., and C. A. Rosen. 1990. Suppression of signals required for activation of transcription factor NF-kB in cells constitutively expressing the HTLV-1 tax protein. New Biol. 2:894-902.

47. Rushlow, C. A., K. Han, J. L. Manley, and M. Levine. 1989. The graded distribution of the dorsal morphogen is initiated by selective nuclear transport in drosophila. Cell 59:1165-1177.

48. Sadowski, I., and M. Ptashne. 1989. A vector for expressing GAL4(1-47) fusions in mammalian cells. Nucleic Acids Res. 17:7539.

49. Saiki, R. K., D. H. Gelfand, S. Stofel, S. J. Scharf, R. Higuchi, G. T. Horn, K. B. Mullis, and H. A. Ehrlich. 1988. Primerdirected enzymatic amplification of DNA with a thermostabile DNA polymerase. Science 239:487-491.

50. Saiki, R. K., S. Scharf, F. Faloona, K. B. Mullis, G. T. Horn, H. A. Ehrlich, and N. Arnheim. 1985. Enzymatic amplification of $\beta$-globin genomic sequences and restriction site analysis for diagnosis of sickle cell anemia. Science 235:1350-1354.

51. Sanger, F., S. Nicklen, and A. R. Coulson. 1977. DNA sequencing with chain-terminating inhibitors. Proc. Natl. Acad. Sci. USA 74:5463-5467.

52. Seiki, M., J.-I. Inoue, T. Takeda, and M. Yoshida. 1986. Direct evidence that $\mathrm{p} 40 \mathrm{x}$ of human T-cell leukemia virus type $\mathrm{I}$ is a trans-acting transcriptional activator. EMBO J. 5:561-565.

53. Short, J. M., J. M. Fernandez, J. A. Sorge, and W. D. Huse. 1988. Lambda Zap: a bacteriophage lambda expression vector with in vivo excision properties. Nucleic Acids Res. 16:75837600.

54. Sodroski, J., C. A. Rosen, W. C. Goh, and W. Haseltine. 1984. A transcriptional activator protein encoded by the c-lor region of the human T-cell leukemia virus. Science 226:61-65.

55. Steward, R. 1987. Dorsasl, an embryonic polarity gene in drosophila is homologous to the vertebrate proto-oncogene, c-rel. Science 238:692-694.

56. Steward, R. 1989. Relocalization of the dorsal protein from the cytoplasm to the nucleus correlates with its function. Cell 59:1179-1188.

57. Struhl, K. 1988. The Jun oncoprotein, a vertebrate transcription factor, activates transcription in yeast. Nature (London) 332: 649.

58. Urban, M., R. Schreck, and P. Baeuerle. 1991. NF-kB contacts DNA by a heterodimer of the p50 and p65 subunit. EMBO J. 10:1817-1825.

59. Urban, M. B., and P. A. Baeuerle. 1990. The $65-\mathrm{kD}$ subunit of NF-kappa-B is a receptor for I-kB and a modulator of DNAbinding specificity. Genes Dev. 4:1975-1984. 
60. Urban, M. B., R. Schreck, and P. A. Baeuerle. 1991. NFkappa-B contacts DNA by a heterodimer of the p50 and p65 subunit. EMBO J. 10:1817-1825.

61. Whitlock, C. A., and O. N. Witte. 1982. Long-term culture of B lymphocytes and their precursors from murine bone marrow. Proc. Natl. Acad. Sci. USA 79:3608.
62. Williams, T., A. Admon, B. Luscher, and R. Tijan. 1988. Cloning and expression of AP-2, a cell-type-specific transcription factor that activates inducible enhancer elements. Genes Dev. 2:1557.

63. Ziff, E. B. 1990 . Transcription factors: a new family gathers at the cAMP response site. Trends Genet. 6:69-72. 